\title{
Sputum expectoration as a useful non-invasive alternative to bronchoalveolar lavage for collecting human alveolar macrophages in tuberculosis research
}

\author{
Li M. Fu ${ }^{1,}$, Charles D. Sohaskey ${ }^{1}$, Brenda Jones ${ }^{2}$ \\ ${ }^{1}$ Research and Development, Veterans Affairs Healthcare System, Long Beach, California \\ ${ }^{2}$ Keck School of Medicine, University of Southern California, Los Angeles, CA
}

Email address:

lifu@patcar.org(L. M. Fu)

To cite this article:

Li M. Fu, Charles D. Sohaskey, Brenda Jones. Sputum Expectoration as a Useful non-Invasive Alternative to Bronchoalveolar Lavage for Collecting Human Alveolar Macrophages in Tuberculosis Research. Science Journal Of Clinical Medicine. Vol. 2, No. 3, 2013 , pp. $92-97$. doi: $10.11648 /$ j.sjcm.20130203.16

\begin{abstract}
As tuberculosis (TB) still claims millions of lives in the world, more research should be directed toward understanding this disease and finding a more effective solution. In vivo human alveolar macrophages are an important focus in TB research since the causative agent, Mycobacterium tuberculosis, can be harbored within these cells in an inactive form for a long time before reactivation. To date, TB researchers collect alveolar macrophage samples using a special clinical procedure known as bronchoalveolar lavage (BAL) in which a bronchoscope is inserted into the lung and the cell samples are collected after being washed out with fluid. BAL is an invasive procedure and its use for a clinical study would often meet with practical constraints and the patient's psychological resistance. These concerns led us to seek a non-invasive approach. In the present study, we collected alveolar macrophage samples from patients with active tuberculosis using a simple sputum expectoration procedure and used the samples to assess the gene expression activities of these immune cells. In this work, we made contributions in two aspects. This study is the first to demonstrate that sputum expectoration is a useful non-invasive alternative for collecting alveolar macrophages whose gene activities would serve to monitor the disease activity. In addition, we analyzed the gene expression of alveolar macrophages in the in vivo samples obtained from TB patients, in contrast to related work where gene expression was profiled on alveolar macrophages collected from non-TB patients and then infected with Mycobacterium tuberculosis in vitro.
\end{abstract}

Keywords: Tuberculosis, Alveolar Macrophage, Sputum Expectoration, Bronchoalveolar Lavage, Gene Expression

\section{Introduction}

Tuberculosis (TB) remains a leading infectious disease of global threat in public health. It causes about two million human deaths per year. According to the statistics published by WHO, over one-third of the world's population now harbors the TB bacterium in their bodies and new infections occur at a rate of one per second. One in ten latent infections will progress to active TB disease with a $50 \%$ of mortality rate if untreated. Active TB can be discriminated from latent $\mathrm{TB}$ by the clinical picture or using a special technique such as plasma proteomic profiling [1]. Unfortunately, current clinical technology does not allow clinicians to predict who among the latent TB population will develop active TB disease. An important research issue is to identify a biomarker for detection of active TB in an early stage when the sputum smear is still negative.

The initial encounter of a host with M. tuberculosis (Mtb) involves phagocytosis of the bacteria by macrophages in the highly aerobic environment of the alveoli. This is followed by replication of the bacteria within inactivated macrophages[2, 3]. Organized granulomas are formed as delayed type hypersensitivity develops and the number of bacteria plateaus. The transcriptional response of macrophages during infection with Mtb has been analyzed [4-7]. An in vitro study of the human THP-1 cell line found that there was an increase in the transcription of a host of cytokines and chemokines [5]. An analysis of human macrophages collected from peripheral blood and infected by $\mathrm{Mtb}$ showed induction of cytokines, chemokines, ribosomal proteins, and the protein for interferon signaling (Stat-1) [6]. The number of genes responsive to Mtb 
infection can be further increased by the addition of IFN- $\alpha$ to activate the macrophages [4].

The study by Thuong et al. showed that the gene expression profiles obtained from macrophages were not entirely similar among three clinical phenotypes of TB, including latent, pulmonary, and meningeal TB, and identified a macrophage gene ccll as a gene involved in human host susceptibility to TB [7]. The results suggest that there exists variation in macrophage resistance against TB bacilli due to genetic differences across individuals and it may be linked to clinical resistance against TB. However, the findings of this study are based on the gene expression profiles of peripheral blood monocyte-derived macrophages rather than alveolar macrophages, which are the front line in contact with the pathogen in pulmonary TB.

In pulmonary tuberculosis, alveolar macrophages have been studied with respect to their roles in host-pathogen interaction and host immunity. For instance, the predominance of low-density alveolar macrophages in TB patients is a manifestation of the pathophysiological response of the lung to TB infection [8]. In addition, alveolar macrophages from TB patients exhibit reduced ability for inhibiting the growth of Mtb [9]. Since alveolar macrophages reside in the lung, there is no simple way to get the samples. Conventionally, alveolar macrophage samples are obtained by means of a specific clinical procedure known as Brochoalveolar lavage (BAL), in which a bronchoscope is passed into the lung, and then sterile saline solution is introduced into some bronchial trees and recollected for examination. BAL is an invasive procedure and requires doctor's supervision. For conducting a clinical study, experimental subjects would feel uncomfortable with BAL more or less because of psychological resistance. Moreover, BAL is not applicable when the facilities for performing bronchoscopy are lacking, as is often the case in a third-world country where TB is prevalent. To facilitate scientific research, it is motivated to explore a non-invasive procedure for collecting alveolar macrophage samples.

In the present work, we delineated a useful protocol consisting of the process of collecting alveolar macrophages using a simple non-invasive procedure referred to as sputum expectoration and the process of analyzing the gene expression of the sample thus obtained with respect to TB activity. The proposed research protocol was demonstrated and validated using clinical samples obtained from TB patients at a hospital affiliated with University of Southern California.

\section{Materials and Method}

A 10cc expectorated sputum sample was collected from each of two patients diagnosed as tuberculosis with sputum-smear $(+)$ and culture $(+)$ by respiratory therapist at the University of Southern California Medical Center under the research protocol approved by the Institutional Review Board (IRB) at the University. The presence of alveolar macrophages in the sputum sample was confirmed under a microscope (see in Figure 1). THP-1 cells were grown in RPMI 1640 with $10 \%$ fetal calf serum.

\subsection{Sample Handling and RNA Extraction}

Sputum samples were snap frozen on dry ice and stored at $-80^{\circ} \mathrm{C}$. The sputum was quickly thawed by the addition of approximately equal volume of warm PBS. Cells were pelleted by centrifuging for $5 \mathrm{~min}(500 \mathrm{~g})$ and immediately lysed with the addition of $500 \mathrm{ml}$ of Trizol (InVitrogen, Carlsbad, CA). RNA was isolated using the Direct-zol RNA MiniPrep kit (Zymo Research). Samples were treated with DNAse on column and eluted in $20 \mu \mathrm{l}$ of water. cDNA was synthesized with a RETROscript kit (Life Technologies, Grand Island, NY).

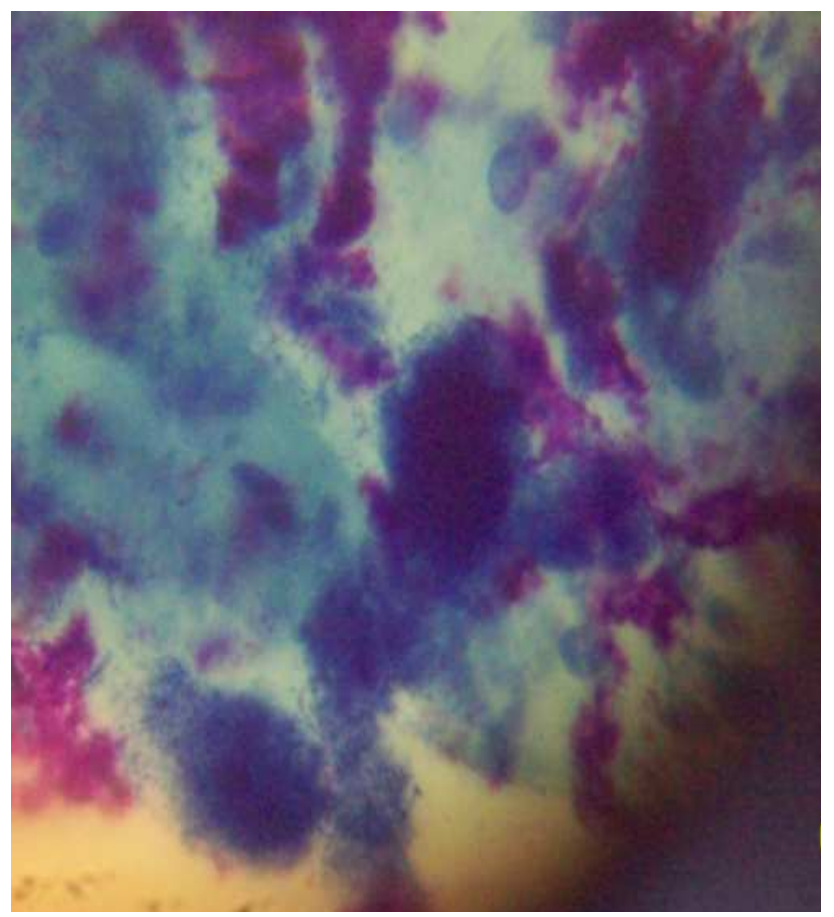

Figure 1. Two macrophages mixed with several squamous epithelial cells seen in this field when an expectorated sputum sample was processed using Giemsa Stain and visualized under a microscope (400x). The squamous epithelial cells came from the contamination from the upper airway.

\subsection{Gene Selection and Polymerase Chain Reaction}

We selected 12 genes for a PCR study, their products including IL-8, RANTES, MIP-1 $\alpha$, MMP-9, Stat-1, P47-PHOX, glutaredoxin, cathepsin K, NF-kB, TCR6, ATP10A, and CXCL10. These genes are selected because they are related to the immune system or they are up-regulated in human macrophages following TB infection [6]. More specifically, IL-8, RANTES, MIP-1 $\alpha$, and CXCL10.are cytokines; Stat-1 mediates interferon signaling; 
TCR6 is a T cell receptor gene; cathepsin $\mathrm{K}$ is an immune peptide protective against TB [10].

Quantitative PCR was performed with the Brilliant SYBR Green QPCR Master Mix kit (Stratagene, La Jolla, CA). Each reaction was performed in a volume of $50 \mu \mathrm{l}$, which consisted of $25 \mu \mathrm{l}$ of $2 \mathrm{X}$ master mix, $5 \mu \mathrm{l}$ of cDNA and both primers at a concentration of $0.1 \mu \mathrm{M}$. Amplification was performed on an ICycler (Bio-Rad, Hercules CA). An initial denaturation of $10 \mathrm{~min}$ at $95^{\circ} \mathrm{C}$ was performed followed by 30 cycles of $95^{\circ} \mathrm{C}$ for $30 \mathrm{sec}$ and $68^{\circ} \mathrm{C}$ for $1 \mathrm{~min}$. This was extended by $7 \mathrm{~min}$ at $68^{\circ} \mathrm{C}$ and a melting curve analysis performed. A control of cDNA without reverse transcriptase was included. An additional cDNA sample was diluted 1:10 to create a standard curve measuring GAPDH. Samples were run on a $2 \%$ agarose gel to verify that only a single band was produced. Each sample was amplified in triplicate. We used primers provided online (http://pga.mgh.harvard.edu/primerbank/)

\section{Gene expression with GAPDH as the standard}

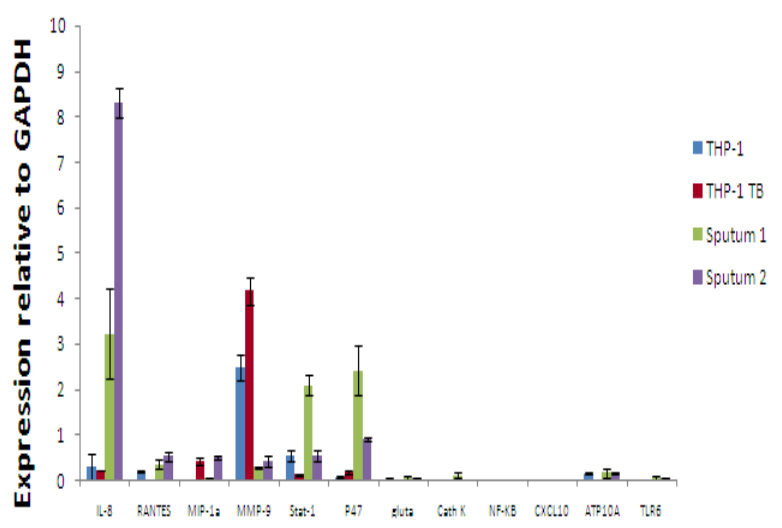

Figure 2. A parallel comparison of the expression levels of the 12 selected genes among the four study groups. The expression level is standardized against that of GAPDH for each group in order to remove the effect due to variation in macrophage cell numbers.

\section{Results}

The objective of the experiment in this study is to determine whether the alveolar macrophage samples collected through sputum expectoration in patients with active tuberculosis disease exhibit meaningful changes in gene expression in consistence with the in vitro model and the literature. The study recorded the macrophage gene expression level of each selected gene in four different types of sample: THP-1 cell without TB infection, THP-1 cells infected with $\mathrm{Mtb}$, the sputum samples collected from the first and second active-TB patients. Each gene expression level was measured three times (triplicate) by Q-PCR. The summary of the average gene expression levels over the triplicate measurements standardized against GAPDH is shown in Table 2, and the overall result is displayed as a side-by-side bar graph in Figure, which allows a parallel comparison of the expression levels of the 12 selected genes among the four groups. The expression level is standardized against that of GAPDH for each group in order to remove the effect due to variation in the quantity of macrophage cells in different samples. To determine genes differentially expressed between normal cells and TB-infected cells, we used the results from the in vitro analysis based on the THP-1 cells. Then we evaluated the results based on the in vivo samples collected from active-TB patients.

Up-regulated genes in response to $\mathrm{TB}$ infection are identified by comparing the expressions of selected genes between the THP-1 cells with and without TB infection. First, we applied the 1.5 fold criterion, and then the statistical test (t-test, two-tailored, $\alpha=0.05$ ) to determine significance. There are four up-regulated genes based on the fold criterion: MIP- $1 \alpha$, MMP-9, P47-PHOX, and NF- $\kappa B$. In particular, MIP-1 $\alpha$ is highly up-regulated with roughly a 100 -fold increase in expression, attributable to TB infection. The difference in the gene expression level before and after TB infection is statistically significant at the $5 \%$ significance level for three genes: MIP-1 $\alpha(\mathrm{P}=$ 0.00037), MMP-9 ( $\mathrm{P}=0.00237)$, and P47-PHOX ( $\mathrm{P}=$ $0.01017)$. The three identified genes with statistical significance would serve as potential gene biomarkers indicative of TB infection. The up-regulation of these three genes agrees with the findings in the literature as discussed later.

Then we analyzed the expression of the identified genes in the sputum samples containing human alveolar macro phages that were collected from TB patients. The alveolar macrophages of the first and second TB patients showed a more than 10-fold increase in gene expression relative to TB-negative THP-1 cells for the genes MIP- $1 \alpha$ and P47-PHOX, but not MMP-9. The results indicate that sputum expectoration is a useful approach for collection of alveolar macrophages encoding disease activity in the context of tuberculosis, and a non-invasive clinical procedure will be instrumental to help research and clinical investigation for this disease.

\section{Discussion}

MIP-1 $\alpha$ (macrophage inflammatory protein 1 alpha) is a cytokine that plays a role in inflammatory responses in macrophages infected by mycobacteria [11] through binding to the chemokine receptors such as CCR1, which mediate the recruitment of immune cells to the inflammatory site. Thus, the expression of MIP- $1 \alpha$ reflects the active disease process sensed by macrophages and could be a good indicator when the intracellular TB bacilli turn from the persistent into active state. MIP- $1 \alpha$ exhibited the highest fold increase relative to the negative 


\section{Gene expression with GAPDH as the standard}

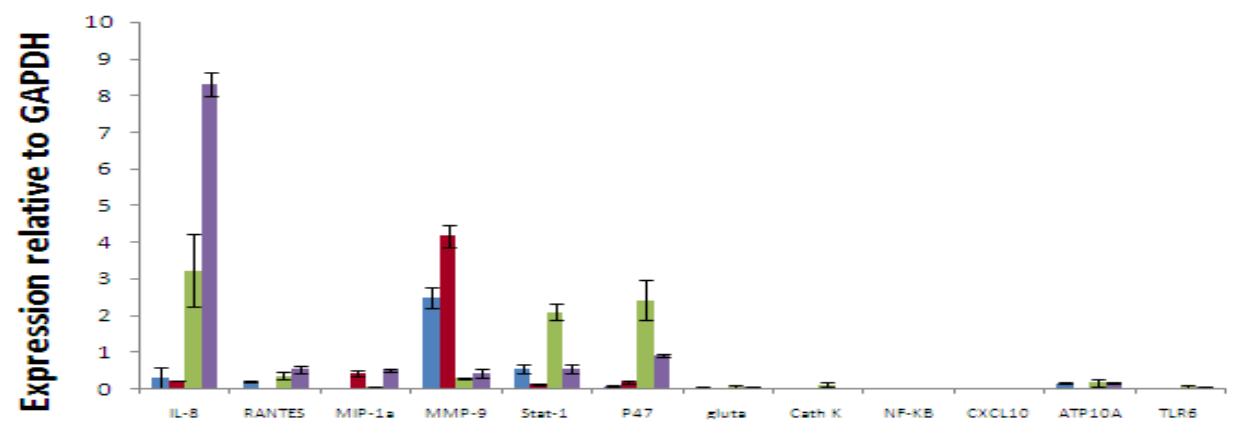

Figure 3. A parallel comparison of the expression levels of the 12 selected genes among the four study groups. The expression level is standardized against that of GAPDH for each group in order to remove the effect due to variation in macrophage cell numbers.

Table 1. The primers for PCR used in this study.

\begin{tabular}{|c|c|c|c|}
\hline Gene & & Primer (5'--3') & Length \\
\hline \multirow[t]{2}{*}{ IL-8 } & Forward & ACTGAGAGTGATTGAGAGTGGAC & 23 \\
\hline & Reverse & AACCCTCTGCACCCAGTTTTC & 21 \\
\hline \multirow[t]{2}{*}{ RANTES } & Forward & CGGCCTCAGACAAAGGGTC & 19 \\
\hline & Reverse & TTCCCGTAAACTTTCTCGCAG & 21 \\
\hline \multirow[t]{2}{*}{ CCL3 (MIP-1a) } & Forward & AGTTCTCTGCATCACTTGCTG & 21 \\
\hline & Reverse & CGGCTTCGCTTGGTTAGGAA & 20 \\
\hline \multirow[t]{2}{*}{ MMP-9 } & Forward & AGACGGGTATCCCTTCGACG & 20 \\
\hline & Reverse & AAACCGAGTTGGAACCACGAC & 21 \\
\hline \multirow[t]{2}{*}{ Stat-1 } & Forward & ATGTCTCAGTGGTACGAACTTCA & 23 \\
\hline & Reverse & TGCTCCAGGAATTTTGAGTCAAG & 23 \\
\hline \multirow[t]{2}{*}{ P47 (phox) } & Forward & CAAGAGTACCGCGACAGACAT & 21 \\
\hline & Reverse & AGGTCTTCTCGTAGTTGGCAAT & 22 \\
\hline \multirow[t]{2}{*}{ cathepsin $\mathrm{K}$} & Forward & ACTCAAAGTACCCCTGTCTCAT & 22 \\
\hline & Reverse & GGGCTCTACCTTCCCATTCTG & 21 \\
\hline \multirow[t]{2}{*}{ NF-kB } & Forward & ACTACCTGGTGCCTCTAGTGA & 21 \\
\hline & Reverse & TTTGACCTGAGGGTAAGACTTCT & 23 \\
\hline \multirow[t]{2}{*}{ CXCL10-c-X-c Motif } & Forward & GTGGCATTCAAGGAGTACCTC & 21 \\
\hline & Reverse & TGATGGCCTTCGATTCTGGATT & 22 \\
\hline \multirow[t]{2}{*}{ ATP10A } & Forward & GGGAGACTTTGTGCGTCTTC & 20 \\
\hline & Reverse & CGATGTGGCATAGCCCGTC & 19 \\
\hline \multirow[t]{2}{*}{ TLR6 } & Forward & TTCTCCGACGGAAATGAATTTGC & 23 \\
\hline & Reverse & CAGCGGTAGGTCTTTTGGAAC & 21 \\
\hline \multirow[t]{2}{*}{ GAPDH } & Forward & CCATGAGAAGTATGACAACAGCC & 23 \\
\hline & Reverse & GGGTGCTAAGCAGTTGGTG & 19 \\
\hline \multirow[t]{2}{*}{ Glutaredoxin } & Forward & CCCATCAAACAAGGGCTTCTG & 21 \\
\hline & Reverse & TGAATCTCGTTAGTGTGGTTGG & 22 \\
\hline \multirow[t]{2}{*}{ 18S RNA } & Forward & CAGGATTGACAGATTGATAGCTCT & 24 \\
\hline & Reverse & GAGTCTCGTTCGTTATCGGAATTA & 24 \\
\hline
\end{tabular}


Table 2. The gene expression level of each selected gene standardized against GAPDH in the four study groups: THP-1 cells without infection, THP-1 cells infected with M. tuberculosis (THP-1 TB), the sputum sample containing human alveolar macrophages from the first TB patient (HAM TB-1) and that from the second TB patient (HAM TB-2).

\begin{tabular}{ccccccccccccc}
\hline & IL-8 & $\begin{array}{c}\text { RANT } \\
\text { ES }\end{array}$ & MIP-1a & MMP-9 & Stat-1 & P47 & gluta & Cath K & NF-KB & $\begin{array}{c}\text { CXCL } \\
\text { 10 }\end{array}$ & $\begin{array}{c}\text { ATP10 } \\
\text { A }\end{array}$ & TLR6 \\
\hline \multirow{2}{*}{ THP-1 } & $2.99 \mathrm{E}-0$ & $1.95 \mathrm{E}-0$ & $3.58 \mathrm{E}-0$ & $2.49 \mathrm{E}+0$ & $5.43 \mathrm{E}-0$ & $6.69 \mathrm{E}-0$ & $2.82 \mathrm{E}-0$ & $7.23 \mathrm{E}-0$ & $1.54 \mathrm{E}-0$ & NA & $1.55 \mathrm{E}-0$ & $2.00 \mathrm{E}-0$ \\
& 1 & 1 & 3 & 0 & 1 & 2 & 2 & 3 & 4 & 1 & 2 \\
THP-1 TB & $2.14 \mathrm{E}-0$ & $2.45 \mathrm{E}-0$ & $4.28 \mathrm{E}-0$ & $4.18 \mathrm{E}+0$ & $1.25 \mathrm{E}-0$ & $1.85 \mathrm{E}-0$ & $3.45 \mathrm{E}-0$ & $7.00 \mathrm{E}-0$ & $3.73 \mathrm{E}-0$ & $1.06 \mathrm{E}-0$ & $2.12 \mathrm{E}-0$ & $1.61 \mathrm{E}-0$ \\
& 1 & 2 & 1 & 0 & 1 & 1 & 3 & 4 & 4 & 6 & 3 & 3 \\
HAM & $3.23 \mathrm{E}+0$ & $3.52 \mathrm{E}-0$ & $6.57 \mathrm{E}-0$ & $2.89 \mathrm{E}-0$ & $2.10 \mathrm{E}+0$ & $2.41 \mathrm{E}+0$ & $5.72 \mathrm{E}-0$ & $1.13 \mathrm{E}-0$ & $2.89 \mathrm{E}-0$ & $1.08 \mathrm{E}-0$ & $1.61 \mathrm{E}-0$ & $5.88 \mathrm{E}-0$ \\
TB-1 & 0 & 1 & 2 & 1 & 0 & 0 & 2 & 1 & 3 & 2 & 1 & 2 \\
HAM & $8.32 \mathrm{E}+0$ & $5.41 \mathrm{E}-0$ & $4.97 \mathrm{E}-0$ & $4.31 \mathrm{E}-0$ & $5.37 \mathrm{E}-0$ & $8.99 \mathrm{E}-0$ & $5.40 \mathrm{E}-0$ & $1.87 \mathrm{E}-0$ & $5.51 \mathrm{E}-0$ & $3.77 \mathrm{E}-0$ & $1.62 \mathrm{E}-0$ & $4.76 \mathrm{E}-0$ \\
TB-2 & 0 & 1 & 1 & 1 & 1 & 1 & 2 & 2 & 3 & 3 & 1 & 2 \\
\hline
\end{tabular}

control among all the genes studied and would serve as a sensitive index for monitoring the disease activity.

MMP (matrix metalloproteinase) family is involved in the breakdown of extracellular matrix in both normal physiological and disease processes, ranging from embryonic development to cancer-associated tissue remodeling. MMP-9 is a collagenase expressed in macrophages and is found to be elevated when the cells are infected with Mtb. However, MMP-9 has a late response compared with MIP-1 $\alpha$ [12], which might explain in part why the in vivo sputum samples from active TB failed to show up-regulation on MMP-9.

P47-PHOX (neutrophil cytosolic factor 1) is the cytosolic subunit of the multi-protein complex called NADPH oxidase. The induction of p47-phox by Mtb in macrophages is linked to oxidative burst [6], which results in the production of various highly reactive oxygen species capable of killing bacteria engulfed by the macrophage. Therefore, the expression pattern of p47-phox is likely to reflect the activity level of TB infection, and could serve as a good biomarker for the disease.

In the literature, both MIP- $1 \alpha$ and MMP-9 are up-regulated in response to $M$. tuberculosis infection in THP-1 cells [5] as well as in MDMs (human monocyte-derived macrophages) [6]. Compared with THP-1 cells, MDMs are more similar to in vivo macrophages and would serve as a better model for studying TB in a clinical setting. However, it is not clear to what extent the in vitro MDMs can simulate in vivo alveolar macrophages in response to TB infection. The simulation of an in vivo environment is complicated by presence of many lurking variables. Thus, it is important to validate a biomarker by testing it against the in vivo sample, as shown in the present work. One previous study used the blood sample taken from TB patients and analyzed the gene expression of the derived MDMs against TB-negative normal subjects, and identified a TB susceptibility gene [7]. In this work, the gene expression pattern would capture the host genetic difference rather than direct in vivo host-pathogen interaction within macrophages since Mtb does not invade peripheral blood monocytes unless in a disseminated bacteremia case.

Similar to MDMs, alveolar macrophages are influenced by host genetics and systemic immune response, but unlike MDMs, alveolar macrophages could capture in vivo host-pathogen interaction. How human alveolar macrophages respond to pulmonary Mtb is an important research question. A study showed that alveolar macrophages recovered from TB patients by BAL on an average have a lower density than those from normal subjects [8], suggesting a change adapted to TB infection in these cells. In another study, alveolar macrophages were collected using BAL and infected with Mtb followed by gene expression analysis [13]. Our study is different from related work in two aspects. First, we used sputum expectoration instead of BAL for collecting alveolar macrophages. Secondly, we analyzed the gene expression of alveolar macrophages in the in vivo samples obtained from TB patients, whereas in related work, gene expression was profiled on alveolar macrophages collected from non-TB patients and then infected with Mtb in vitro. Unlike bronchoalveolar lavage, sputum expectoration is a non-invasive technique for obtaining a sample of human alveolar macrophages. The present work showed that a 10 $\mathrm{cc}$ of expectorated sputum specimen contains enough human alveolar macrophages for gene activity analysis.

\section{Conclusions}

Recent research has highlighted the need to improve our understanding about the role of human alveolar macrophages in active and latent tuberculosis. However, there are two limitations with current research methodology in this area. First, alveolar macrophages were collected using an invasive procedure known as bronchoalveolar lavage, which would often keep human subjects refraining from participating in a clinical study often because of psychological resistance. Secondly, the gene expression analysis was performed on alveolar macrophages collected from non-TB patients and infected with M. tuberculosis. To overcome the limitations, we present a useful protocol to 
collect alveolar macrophages using a non-invasive procedure called sputum expectoration and to analyze the sample so-obtained. Then, we demonstrate that sputum expectoration is a viable non-invasive alternative for collecting alveolar macrophages whose gene activities would serve to monitor the disease activity. In contrast to related work, we analyzed the gene expression of alveolar macrophages in the in vivo samples obtained from TB patients. Thus our approach will more accurately identify what happens concerning in vivo host-pathogen interaction with tuberculosis.

\section{Acknowledgment}

Edward $\mathrm{Fu}$ helped the project by determining the differential cell counts of a sputum sample under a microscope.

\section{References}

[1] Sandhu G, Battaglia F, Ely B K, et al. Discriminating active from latent tuberculosis in patients presenting to community clinics. PLoS One 2012; 7(5): e38080.

[2] Dannenberg A M, Jr., Pathogenesis of pulmonary tuberculosis: an interplay of tissue-damaging and macrophage-activating immune responses-dual mechanisms that control bacillary multiplication, in Tuberculosis: Pathogenesis, protection and control, B.R. Bloom, Editor. 1993, American Society for Microbiology: Washington, D.C. p. $459-483$.

[3] Lauzardo M, Ashkin D. Phthisiology at the dawn of the new century. Chest 2000; 117(5): 1455-73.

[4] Ehrt S, Schnappinger D, Bekiranov S, et al. Reprogramming of the macrophage transcriptome in response to interferon-gamma and Mycobacterium tuberculosis: signaling roles of nitric oxide synthase- 2 and phagocyte oxidase. J Exp Med 2001; 194(8): 1123-40.
[5] Ragno S, Romano M, Howell S, Pappin D J, Jenner P J, Colston M J. Changes in gene expression in macrophages infected with Mycobacterium tuberculosis: a combined transcriptomic and proteomic approach. Immunology 2001; 104(1): 99-108.

[6] Wang J P, Rought S E, Corbeil J, Guiney D G. Gene expression profiling detects patterns of human macrophage responses following Mycobacterium tuberculosis infection. FEMS Immunol Med Microbiol 2003; 39(2): 163-72.

[7] Thuong N T, Dunstan S J, Chau T T, et al. Identification of tuberculosis susceptibility genes with human macrophage gene expression profiles. PLoS Pathog 2008; 4(12): e1000229.

[8] Kuo H P, Yu C T. Alveolar macrophage subpopulations in patients with active pulmonary tuberculosis. Chest 1993; 104(6): 1773-8.

[9] Lennart P H, Eklund D, Larsson M, Welin A, Paues J, Idh J. Alveolar macrophages from patients with tuberculosis exhibit reduced capacity of restricting growth of Mycobacterium tuberculosis: a pilot study of vitamin D stimulation in vitro. Microbiology Discovery 2013, . 1-6. doi: 10.7243/2052-6180-1-6

[10] Nepal R M, Mampe S, Shaffer B, Erickson A H, Bryant P. Cathepsin L maturation and activity is impaired in macrophages harboring $M$. avium and $M$. tuberculosis. Int Immunol 2006; 18(6): 931-9.

[11] Sadek M I, Sada E, Toossi Z, Schwander S K, Rich E A. Chemokines induced by infection of mononuclear phagocytes with mycobacteria and present in lung alveoli during active pulmonary tuberculosis. Am J Respir Cell Mol Biol 1998; 19(3): 513-21.

[12] Chang J C, Wysocki A, Tchou-Wong K M, Moskowitz N, Zhang Y, Rom W N. Effect of Mycobacterium tuberculosis and its components on macrophages and the release of matrix metalloproteinases. Thorax 1996; 51(3): 306-11.

[13] Silver R F, Walrath J, Lee H, et al. Human alveolar macrophage gene responses to Mycobacterium tuberculosis strains H37Ra and H37Rv. Am J Respir Cell Mol Biol 2009; 40(4): 491-504. 